\title{
EXTRAÇÃO SUPERCRÍTICA COM UTILIZAÇÃO DE MODIFICADORES E CARACTERIZAÇÃO A PARTIR DA SEMENTE DE CUMARU (DIPTERYX ODORATA)
}

\author{
J. de C. LIMA $^{1}$, L. F. PINTO ${ }^{1}$, W. M. GIUFRIDA ${ }^{1}$, L. dos S. FREITAS ${ }^{2}$, L. CARDOZO- \\ FILHO $^{1}$.
}

${ }^{1}$ Universidade Estadual de Maringá - Departamento de Engenharia Química

${ }^{2}$ Universidade Federal de Sergipe - Departamento de Química

E-mail para contato: je.clima@hotmail.com

\begin{abstract}
RESUMO - Este trabalho tem como objetivo a extração e quantificação de compostos bioativos da semente do cumaru por meio de extração convencional (soxhlet) e supercrítica utilizando dióxido de carbono e solventes modificadores. As extrações supercríticas foram realizadas numa faixa de pressão de 180 - 220 bar e temperatura de $60-80^{\circ} \mathrm{C}$. O planejamento experimental foi definido a partir do design fatorial fracionado (método Taguchi). Os extratos foram analisados por cromatografia liquida (HPLC) e gasosa com espectrofotômetro de massas (GCMS). A extração da cumarina utilizando o dióxido de carbono e modificados apresentou melhor seletividade e rendimentos em relação à extração convencional.
\end{abstract}

\section{INTRODUÇÃO}

A cumarina é um composto formado por um anel aromático fundido em um anel lactona com potencial para utilização de herbicida (Oliveros-bastidas et al., 2013), perfumes, cosméticos, cigarros (Sullivan, 1982), anti-inflamatório (Leal et al., 2000) além de fins medicinais, como na prevenção e tratamento de edemas consequentes da radioterapia e vários tipos de câncer (Grötz et al., 2001; Mohler et al., 1994; Thornes et al., 1994).

Foi isolada de diferentes espécies (Mitra et al., 2009; Oliveira et al., 2013; Rodrigues et al., 2008) e é amplamente distribuída nas sementes de Cumaru (Dipteryx odorata), justificando o nome da espécie, tendo sida isolada também desta semente em pesquisa anterior, por meio de extração com Soxhlet (Sullivan, 1982). No entanto, este tipo de extração apresenta desvantagens como grande utilização de solventes orgânicos, demanda de longos períodos de extração e o fato de não ser seletivo, obtendo muitos analitos como subproduto.

Neste sentido surge a possibilidade de extração com fluido supercrítico (EFS), cuja pressão e temperatura se encontram acima do ponto crítico. Acima da temperatura crítica o solvente não volta a ser liquefeito, no entanto, com o aumento da pressão, a densidade se aproxima a do estado líquido e o poder de solvatação é elevada. 
O dióxido de carbono tem sido o preferencialmente utilizado neste tipo de extração, por apresentar propriedades críticas relativamente baixas, baixo custo, não ser tóxico, não inflamável, ser inofensivo ao meio ambiente e prontamente disponível no mercado com alto grau de pureza (King and Bott, 1993). No entanto, o dióxido de carbono apresenta a desvantagem de ser altamente apolar, dificultando a extração de analitos polares ou levemente polares, como é o caso da cumarina. $\mathrm{O}$ uso de pequenas quantidades de solventes orgânicos como modificadores vêm revertendo este quadro, mantendo os benefícios da EFS.

O principal objetivo desta pesquisa é avaliar a extração da cumarina a partir da semente de cumaru por meio da extração supercrítica com o uso de pequenas porcentagens de solventes orgânicos (modificadores), como o acetato de etila, diclorometano e etanol. Avaliar a seletividade e o rendimento do componente alvo utilizando Cromatografia Liquida de Alta Eficiência (CLAE) e obter perfil de ácidos graxos livres utilizando Cromatografia Gasosa equipado com espectrômetro de massas (CG-EM).

\section{MATERIAL E MÉTODO}

\subsection{Matriz vegetal.}

A semente de cumaru foi adquirida na Oriental Central Comércio Ltda, no Estado do Pará, Brasil, em junho de 2013. Foi utilizado um processador de uso doméstico afim de obter um diâmetro de partícula da semente entre 1 e $2 \mathrm{~mm}$. Após, o material foi armazenado sob refrigeração até o inicio das extrações.

\subsection{Extração com Soxhlet.}

Para as extrações utilizando Soxhlet foram utilizados em média 20 gramas da semente, $350 \mathrm{~mL}$ de hexano como solvente na primeira extração e $350 \mathrm{~mL}$ de etanol na segunda, na temperatura de $45^{\circ} \mathrm{C}$, durante 48 horas. Os rendimentos foram calculados de acordo com a massa inicial da amostra.

\subsection{Extração com Fluido Supercrítico.}

O aparato em escala laboratorial consisti em um cilindro de dióxido de carbono, 99,9\% de pureza obtido da White Martins S.A. (Brasil), duas bombas seringa (ISCO, Model 500D), sendo que uma foi abastecida com o dióxido de carbono (bomba A) e outra com o solvente orgânico (modificador) (bomba B), dois banhos termostáticos e um extrator cilíndrico com $163 \mathrm{~mL}$ de volume interno.

As sementes foram adicionadas no extrator aquecidas a temperaturas variando entre 60 e $80^{\circ} \mathrm{C}$. O extrator foi então pressurizado com o dióxido de carbono por meio da bomba $\mathrm{A}$ e após, adicionado o modificador pela bomba B. O sistema permaneceu em equilíbrio durante 20 minutos. Em seguida, uma válvula de controle da vazão de extração foi aquecida a $80^{\circ} \mathrm{C}$, a fim de evitar o congelamento do $\mathrm{CO}_{2}$ e entupimento da mesma. A mesma, foi manuseada para controle do fluxo da extração a uma vazão de $3 \mathrm{~mL} \cdot \mathrm{min}^{-1}$. A cinética de extração foi feita a 
cada intervalo de 20 minutos. Após 140 minutos de extração a válvula foi fechada e o sistema foi despressurizado.

O planejamento experimental foi realizado utilizando uma padronização do modelo fatorial fracionado e arranjos ortogonais, conhecido como Método de Taguchi. Este método possui a vantagem de analisar um número maior de variáveis com realização de menor número de experimentos, obtendo resultados pela combinação de respostas Neste caso o planejamento foi formado por quatro variáveis (tipo de modificador, temperatura, pressão e porcentagem de modificador) e três níveis, totalizando em nove experimentos, realizados em duplicata. Os dados foram avaliados por Análise de Variância

\subsection{Cromatografia Líquida de Alta Eficiência (HPLC).}

As soluções foram preparadas utilizando metanol (40\%) + acetonitrila (60\%) para solubilização das amostras. Foram realizadas injeções de $2 \mu \mathrm{L}$ em um HPLC Varian Polaris Modelo 210, equipado com coluna cromatográfica Luna $5 \mu \mathrm{m}$, fase estacionária C18 (2) 100 $\AA$ ( $150 \mathrm{~mm}$ x 4,6 mm), operando a $30^{\circ} \mathrm{C}$. A fase móvel utilizada foi: $5 \%$ de ácido acético em fase aquosa (fase A) e uma mistura de $60 \%$ acetonitrila e $40 \%$ metanol (fase B), em modo gradiente, conforme indicado na Tabela 1.

Tabela 1 Eluição Gradiente da Fase Móvel.

\begin{tabular}{ccc}
\hline Tempo de corrida (min) & Proporção de Fases A:B & Isocrático (min) \\
\hline 0 & $60: 40$ & 5 \\
10 & $40: 60$ & 0 \\
20 & $10: 90$ & 5 \\
25 & $60: 40$ & 0 \\
\hline
\end{tabular}

O UV-VIS foi utilizado como detector em um comprimento de onda $274 \eta \mathrm{m}$. Os dados foram processados através do software Galaxie.

Foi injetada também uma solução com uma amostra padrão de cumarina (Synth 99,5\% Brasil) a fim de comparação do tempo de retenção. Para a quantificação do composto no extrato foi feita uma curva de calibração da cumarina padrão nas concentrações de 20, 25, 30, 35 e 40 ppm. Para determinação das concentrações da cumarina, referente a cada extração realizada, foi utilizada a equação da reta a partir da área dos picos cromatográficos de cada extrato injetado.

\subsection{Cromatografia Gasosa}

As amostras foram derivatizadas utilizando o BSTFA (Bistrimethylsilyltrifluoroacetamide), onde ocorreu a troca de hidrogênios ativos por um grupo trimetilsilil. Para a sililação $10 \mathrm{mg}$ de cada amostra foram acondicionadas em um vial, sendo acrescentado $25 \mu \mathrm{L}$ do padrão interno (heptadecanoato de metila) e $25 \mu \mathrm{L}$ de BSTFA e diclorometano até completar $1 \mathrm{~mL}$. $\mathrm{O}$ vial foi selado com septo e a amostra foi aquecida uniformemente em banho de areia a $60^{\circ} \mathrm{C}$ por 30 minutos para assegurar que ocorresse a reação completa. 
A quantificação de ácidos graxos foi realizada em um cromatógrafo gasoso equipado com um espectrômetro de massas da marca Shimadzu GCMS-QP2010 Plus, com coluna capilar ZB-5MS (diâmetro interno $0,25 \mathrm{~mm} \times 30 \mathrm{~m}$, espessura do filme $0,25 \mu \mathrm{m}$ ). A temperatura do injetor foi de $280^{\circ} \mathrm{C}$; volume da injeção de $1 \mu \mathrm{L}$, Split 1:20. As condições de operação estão descritas na Tabela 2.

Tabela 2 Condições de Operação GC-MS

\begin{tabular}{lll}
\hline T.C. $\left({ }^{\circ} \mathrm{C}\right) *$ & T. T. $\left({ }^{\circ} \mathrm{C} / \mathrm{min}\right) * *$ & T. P. $(\mathrm{min}) * * *$ \\
\hline 110 & 0 & 2 \\
200 & 8 & 4 \\
210 & 2 & 6 \\
280 & 15 & 0 \\
\hline Fluxo de gás hélio $=1 \mathrm{~mL} /$ min. Pressão constante $=76,9 \mathrm{KPa}$ \\
*T.C. - Temperatura da Coluna \\
**T. T. Taxa de Aumento de Temperatura \\
*** Tempo de Permanência
\end{tabular}

O espectrômetro foi conduzido com o modo de impacto de elétrons a $70 \mathrm{eV}$. A leitura foi efetuada no modo SIM, onde a razão massa/carga monitoradas foram 73 e 74 . A identificação dos ácidos graxos na amostra foi realizada por meio do tempo de retenção da solução padrão, com a confirmação dos fragmentos de massa presentes nos espectros de massa no modo SCAN. A quantificação foi desenvolvida por padronização interna levando em consideração a área e o fator de resposta calculado para cada ácido graxo analisado (Hirschegger et al., 2014; Jacques et al., 2006).

\section{DISCUSSÃO DE RESULTADOS}

\subsection{Rendimento Global em Termos de Massa de Extrato.}

Os resultados obtidos nas extrações supercríticas e com solventes orgânicos estão disponíveis na Tabela 3.

Tabela 3 Planejamento Experimental e Rendimento das Extrações

\begin{tabular}{ccccccccc}
\hline Exp. & Tipo do Modificador & $\mathrm{T}\left({ }^{\circ} \mathrm{C}\right)$ & $\mathrm{P}($ bar $)$ & Modificador\% & $\begin{array}{c}\text { Rendimento do } \\
\text { Extrato }(\%)\end{array}$ & $\begin{array}{c}\text { Cumarina } \\
\text { extrato }(\%)\end{array}$ & $\begin{array}{c}\text { Cumarina } \\
\text { semente }(\%)\end{array}$ & Nomenclatura \\
\hline 1 & Acetato de Etila & 60 & 180 & 1 & $3,39 \pm 0,21$ & 7,19 & 0,26 & A111 \\
2 & Acetato de Etila & 70 & 200 & 3 & $3,27 \pm 0,24$ & 5,27 & 0,12 & A222 \\
3 & Acetato de Etila & 80 & 220 & 5 & $3,85 \pm 0,22$ & 2,07 & 0,09 & A333 \\
4 & Etanol & 60 & 200 & 5 & $4,15 \pm 0,17$ & 11,55 & 0,55 & E123 \\
5 & Etanol & 70 & 220 & 1 & $2,87 \pm 0,04$ & 8,17 & 0,26 & E231 \\
6 & Etanol & 80 & 180 & 3 & $1,93 \pm 0,07$ & 20,92 & 0,44 & E312 \\
7 & Diclorometano & 60 & 220 & 3 & $4,76 \pm 0,22$ & 3,96 & 0,20 & D132 \\
8 & Diclorometano & 70 & 180 & 5 & $2,61 \pm 0,21$ & 11,75 & 0,36 & D213 \\
\hline
\end{tabular}




\begin{tabular}{ccccccccc}
\hline 9 & Diclorometano & 80 & 200 & 1 & $2,33 \pm 0,01$ & 13,10 & 0,34 & D321 \\
10 & Soxhlet com Etanol & 45 & - & - & $46,50 \pm 0,30$ & 0,51 & 0,29 & S_etanol \\
11 & Soxhlet com Hexano & 45 & - & - & $19,13 \pm 0,87$ & 1,43 & 0,34 & S_hexano \\
\hline
\end{tabular}

Observa-se na Tabela 3 que as extrações por Soxhlet resultaram em rendimentos superiores as EFS em termos de massa, principalmente a extração através de etanol, que resultou num rendimento de $46,5 \%$, enquanto as extrações com dióxido de carbono obtiveram um rendimento máximo de $4,76 \%$. Este comportamento pode estar relacionado à diversos fatores, como o tempo de extração superior, recirculação do solvente, e maior afinidade do solvente com os compostos extraídos (Benelli et al., 2010; Gonçalves et al., 2013).

$\mathrm{O}$ experimento utilizando $3 \%$ de diclorometano, menor temperatura $\left(80^{\circ} \mathrm{C}\right)$ e maior pressão $\left(220^{\circ} \mathrm{C}\right)$ apresentou maior rendimento em termos de massa. Este comportamento já era esperado, devido a influencia da densidade do $\mathrm{CO}_{2}$ em seu poder de dissolução, sendo proporcional a pressão com temperatura constante e inversamente proporcional a temperatura quando a pressão é constante, principalmente em zonas próximas ao ponto crítico (King and Bott, 1993). Como a quantidade de modificador utilizada foi de apenas $3 \%$ e o $\mathrm{CO}_{2}$ se encontra em estado gasoso em condições ambientes, o extrato final apresentou-se livre de solventes.

\subsection{Rendimento em termos de porcentagem de Cumarina}

Embora os rendimentos em termos de massa tenham sido elevados na extração com Soxhlet, os rendimentos das extrações com $\mathrm{CO}_{2}$ supercrítico apresentaram maiores seletividade de cumarina, destacando a extração utilizando $3 \%$ de etanol, maior temperatura $\left(80^{\circ} \mathrm{C}\right)$ e menor pressão (180 bar), como observado na Tabela 3.

A utilização de menores pressões aumentam a seletividade, como já havia sido descrito em pesquisas anteriores, visto que o aumento da densidade pode aumentar a solubilidade com outros componentes (Lemos et al., 2012). É possível confirmar esta hipótese observando os rendimentos da extração 7, que apresenta um maior rendimento de massa, no entanto, apresenta uma baixa seletividade $(3,96 \%$ de cumarina no extrato).

\subsection{Análise de Variância}

As variáveis escolhidas foram avaliadas quanto a sua significância por meio da Análise de Variância (ANOVA) e todas foram consideradas influentes no rendimento da extração ( $\mathrm{p}<0,05)$, embora o tipo de modificador utilizado, seguido da pressão, tenha mostrado maior significância.

\subsection{Perfil de Ácidos Graxos Livres}

Na Tabela 4 está disposta a determinação do perfil de ácidos graxos livres para cada amostra. Em todas as condições de extração prevaleceu a presença de ácido oleico (ômega 9) seguido do ácido linoleico (ômega 6). 
A razão $\mathrm{O} / \mathrm{L}$ (ácido oleico/linoleico) presente na Tabela 4 é um indicativo da estabilidade do óleo (Freitas, 2007), que, neste caso, pode ser considerado estável, visto que apresenta similaridade para todas as condições de extração (aproximadamente 2,7).

Tabela 4 Normalização dos ácidos graxos livres

\begin{tabular}{ccccccccccccc}
\hline & A111 & A222 & $\mathbf{A 3 3 3}$ & $\mathbf{D 2 1 3}$ & $\mathbf{D 3 2 1}$ & $\mathbf{D 1 3 2}$ & $\mathbf{E 3 1 2}$ & $\mathbf{E 1 2 3}$ & E231 & Soxhlet Etanol & Soxhlet hexano \\
\hline Ác Láurico & n.d. & n.d. & n.d. & n.d. & n.d. & n.d. & n.d. & n.d. & n.d. & n.d. & n.d. \\
Ác Mirístico & 0,1 & 0,3 & 0,3 & 0,2 & 0,2 & 0,2 & 0,3 & 0,1 & 0,2 & 0,0 & 0,3 \\
Ác Palmitoleico & n.d. & n.d. & n.d. & n.d. & n.d. & n.d. & n.d. & n.d. & n.d. & n.d. & n.d. \\
Ác Palmítico & 12,1 & 12,7 & 12,1 & 11,9 & 12,6 & 11,8 & 13,4 & 11,1 & 12,1 & 11,5 & 12,3 \\
Ác Linoleico & 22,1 & 22,8 & 22,8 & 22,3 & 22,5 & 21,8 & 23,0 & 20,5 & 22,3 & 20,8 & 21,0 \\
Ác Oleico & $\mathbf{5 8 , 2}$ & $\mathbf{5 5 , 7}$ & $\mathbf{5 6 , 0}$ & $\mathbf{5 8 , 3}$ & $\mathbf{5 6 , 8}$ & $\mathbf{5 8 , 6}$ & $\mathbf{5 4 , 6}$ & $\mathbf{6 1 , 7}$ & $\mathbf{5 7 , 9}$ & $\mathbf{5 9 , 8}$ & $\mathbf{5 8 , 8}$ \\
Ác Esteárico & 7,5 & 8,5 & 8,9 & 7,4 & 7,9 & 7,7 & 8,7 & 6,6 & 7,5 & 7,9 & 7,6 \\
Acidez Total & 3,7 & 4,9 & 6,3 & 3,8 & 4,3 & 5,7 & 12,3 & 3,4 & 5,5 & 2,6 & 1,4 \\
O/L & 2,6 & 2,4 & 2,5 & 2,6 & 2,5 & 2,7 & 2,4 & 3,0 & 2,6 & 2,9 & 2,8 \\
\hline
\end{tabular}

\section{CONCLUSÃO}

A metodologia desenvolvida para extração de cumarina a partir da semente de cumaru utilizando a tecnologia supercrítica apresentou resultado satisfatório em relação à extração convencional, tendo em vista a maior seletividade da cumarina, maior rapidez na obtenção do extrato e obtenção de um produto mais limpo, livre de resíduos de solventes orgânicos. Sendo mais indicada para emprego na indústria farmacêutica e de cosméticos, com destaque para o uso de etanol, que apresentou maior rendimento, possui baixo custo e apresenta baixa toxicidade. O perfil de ácidos graxos livres, obtido na caracterização da semente apresentou teores de ácidos insaturados superiores aos saturados, sendo mais uma característica favorável na utilização deste extrato. 


\section{REFERENCIAS}

Benelli, P., Riehl, C. a. S., Smânia Jr., A., Smânia, E.F. a., Ferreira, S.R.S. Bioactive extracts of orange (Citrus sinensis L. Osbeck) pomace obtained by SFE and low pressure techniques: Mathematical modeling and extract composition. J. Supercrit. Fluids 55, 132-141, 2010.

Freitas, L. dos Santos. Desenvolvimento de procedimentos de extração do óleo de semente de uva e caracterização química dos compostos extraídos, 2007.

Gonçalves, R.M., Lemos, C.O.T., Leal, I.C.R., Nakamura, C.V., Cortez, D.A.G., da Silva, E.A., Cabral, V.F., Cardozo-Filho, L. Comparing conventional and supercritical extraction of (-)-mammea $\mathrm{A} / \mathrm{BB}$ and the antioxidant activity of Calophyllum brasiliense extracts.

Molecules 18, 6215-29, 2013.

Grötz, K.A., Wüstenberg, P., Kohnen, R., Zepelin, H.H. Prophylaxis of radiogenic sialadenitis and mucositis by coumarin / troxerutine in patients with head and neck cancer - a prospective , randomized , placebo-controlled, double-blind study 34-39, 2001.

Hirschegger, L., Schober, S., Mittelbach, M. Efficient and sensitive method for the quantification of saturated monoacylglycerols in biodiesel by gas chromatography-mass spectrometry. Eur. J. Lipid Sci. Technol. 116, 89-96, 2014.

Jacques, R.A., Freitas, L. dos S., Peres, V.F., Dariva, C., Oliveira, J.V. de, Caramão, E.B. Chemical composition of mate tea leaves (Ilex paraguariensis): A study of extraction methods. J. Sep. Sci. 29, 2780-2784, 2006.

King, M.B., Bott, T.R. Extraction of Natural Products Using Near-Critical Solvents, 1993.

Leal, L.K., Ferreira, a a, Bezerra, G. a, Matos, F.J., Viana, G.S. Antinociceptive, antiinflammatory and bronchodilator activities of Brazilian medicinal plants containing coumarin: a comparative study. J. Ethnopharmacol. 70, 151-9, 2000.

Lemos, C.O.T., Garcia, V.A.D.S., Gonçalves, R.M., Leal, I.C.R., Siqueira, V.L.D., Filho, L.C., Cabral, V.F. Supercritical extraction of neolignans from Piper regnelli var. pallescens. J. Supercrit. Fluids 71, 64-70, 2012.

Mitra, P., Barman, P.C., Chang, K.S. Coumarin Extraction from Cuscuta reflexa using Supercritical Fluid Carbon Dioxide and Development of an Artificial Neural Network Model to Predict the Coumarin Yield. Food Bioprocess Technol. 4, 737-744, 2009.

Mohler, J.L., Williams, B.T., Thompson, I.M., Marshall, M.E. Coumarin (1,2-benzopyrone) for the treatment of prostatic carcinoma. J. Cancer Res. Clin. Oncol. 120 Suppl, S35-8, 1994.

Oliveira, a. L., Pozza, L.N.L., Santos, D.N., Kamimura, E.S., Vicente, E., Cabral, F. a. Supercritical extraction of coumarin from guaco (Mikania laevigata and Mikania glomerata) for pharmaceutical applications. J. Supercrit. Fluids, 2003. 
Oliveros-bastidas, A.D.J., Demuner, A.J., Barbosa, L.C. de A. Chemical Characterization by GC-MS and Phytotoxic Potential of non-polar and polar fractions of seeds of Dipteryx odorata (Aubl.) Wild. from Venezuelan regions 36, 502-506, 2013.

Rodrigues, R.F., Tashima, A.K., Pereira, R.M.S., Mohamed, R.S., Cabral, F. a. Coumarin solubility and extraction from emburana (Torresea cearensis) seeds with supercritical carbon dioxide. J. Supercrit. Fluids 43, 375-382, 2008.

Sullivan, G., Occurrence of Umbelliferone in the Seeds of Dipteryx odorata ( Aubl .) Willd . J. Agric. Food Chem. 609-610, 1982.

Thornes, R.D., Daly, L., Lynch, G., Breslin, B., Browne, H., Browne, H.Y., Corrigan, T., Daly, P., Edwards, G., Gaffney, E., Henley, J., Healy, T., Keane, F., Lennon, F., Mcmurray, N., Loughlin, S.O., Shine, M., Tanner, A. Treatment with coumarin to prevent or delay recurrence of malignant melanoma 120, 32-34, 1994. 